\title{
Watch out for Abdominal Pain in HIV-AIDS Patients: Abdominal Tuberculosis
}

\section{María Rodríguez-Santiago*, Luis Alvarez-Pérez, José Gaudier-Díaz, Pedro Gil-De Rubio, Natalia Hernández-Cuevas, José Colón-Márquez}

Department of Internal Medicine, University of Puerto Rico, School of Medicine, San Juan, Puerto Rico

Email: ^maria.rodriguez86@upr.edu

How to cite this paper: Rodríguez-Santiago, M., Alvarez-Pérez, L., Gaudier-Díaz, J., Rubio, P.G.-D., Hernández-Cuevas, N. and Colón-Márquez, J. (2020) Watch out for Abdominal Pain in HIV-AIDS Patients: Abdominal Tuberculosis. Case Reports in Clinical Medicine, 9, 392-398.

https://doi.org/10.4236/crcm.2020.912054

Received: November 16, 2020

Accepted: December 26, 2020

Published: December 29, 2020

Copyright $\odot 2020$ by author(s) and Scientific Research Publishing Inc. This work is licensed under the Creative Commons Attribution International License (CC BY 4.0).

http://creativecommons.org/licenses/by/4.0/

\begin{abstract}
Extrapulmonary Tuberculosis (EPTB) is the TB involving organs other than lungs. The diagnosis of EPTB can be difficult, as suspicion is challenging, because it presents with nonspecific clinical features and atypical presentation. Furthermore, patients with HIV may present with fever of unknown origin (FUO) as the only symptom. We present a clinical case of a 49 years old $\mathrm{Pu}$ ertorrican female with HIV-AIDS and no high-risk behavior with an abdominal pain and fever. The investigation confirmed an Abdominal Tuberculosis. This case emphasizes the need to add Abdominal tuberculosis (ATB) within the differential diagnosis and discuss the diagnostic process.
\end{abstract}

\section{Keywords}

Abdominal Pain, Abdominal Tuberculosis (ATB), Diagnosing ATB, HIV/AIDS

\section{Introduction}

Tuberculosis (TB) is a disease caused by Mycobacterium tuberculosis and nontuberculous Mycobacteria (NTM), which are obligate aerobe acid fast bacillus (Rods). It is a slow-growing bacterium, which may take up to six weeks for visible growth. M. tuberculosis is characterized by forming nodule-like strictures called Granulomas, which are an area of inflammation composed of bacteria surrounded by infected macrophages and other layers of immune cells including granulocytes, dendritic cells (DCs), natural killer (NK) cells, and T and B lymphocytes [1]. Extrapulmonary TB (EPTB) is the TB involving organs other than lungs. A patient with both pulmonary and EPTB is considered a case of Pulmonary TB [2]. In HIV-positive patients, EPTB accounts for more than $50 \%$ of all Tuberculosis's cases [3]. The diagnosis of EPTB can be difficult, as suspicion is 
challenging, because it presents with nonspecific clinical features and atypical presentation [4]. Also, anatomical areas are usually difficult to access and radiological features are non-specific, which adds to the difficulty of the diagnosis [5]. EPTB may manifest with constitutional symptoms such as fever, anorexia, weight loss, malaise and fatigue. Patients with HIV may present with fever of unknown origin (FUO) as the only symptom. The proportion of TB in the final diagnosis of FUO was $8 \%-11 \%$ in the 2000s. In fact, in South Korea, TB is automatically within the differential diagnosis when evaluating FUO [4]. According to Sharma et al. (2004), the clinical presentation of Abdominal Tuberculosis (ATB) can be acute, chronic or acute on chronic. The most common clinical presentations are fever, abdominal pain, diarrhea, constipation or alternating pattern of diarrhea and constipation [6]. On Awasthi et al. (2015), all 48 participants with ATB reported abdominal pain, thus being the most common clinical presentation. Therefore, ATB should be within the differential diagnosis in high-risk patients presenting with vague abdominal symptoms [7].

This case in discussion highlights the importance of clinical suspicion and diagnostic methods of ATB in patients with Human Immunodeficiency VirusAcquired Immune Deficiency Syndrome (HIV-AIDS) presenting with abdominal pain. Early recognition of signs and symptoms could prevent worsening of disease, and thus better outcomes pertaining the morbidity and mortality of these patients.

\section{Case Report}

This is the case of a forty-nine years old female with history of Human Immunodeficiency Virus (HIV)/Acquired Immune Deficiency Syndrome (AIDS) (CD4: 149, Viral load: 406) non-compliant with highly active anti-retroviral therapy (HAART) of Bictegravir-Emcitrabine-Tenofovir alafenamide who developed an intermittent stabbing periumbilical and Left Lower Quadrant (LLQ) abdominal pain associated with intermittent episodes of fever, chills, night sweats and general malaisesince three weeks prior to evaluation. The patient denied someone at home with similar symptoms, personal history of Tuberculosis (TB), recent traveling, imprisonment or homelessness. Physical evaluation was pertinent for an underweight female with a Body Mass Index (BMI) $<18.5 \mathrm{~kg} / \mathrm{m}^{2}$ with a LLQ 10 $\times 6 \mathrm{~cm}$ non-mobile, tender to palpation violaceous mass without erythema, warmth or pus drainage (Figure 1). During the second day of hospitalization, patient developed aHerpetic zoster lesion in Left eye treated with topical and intravenous administration of Acyclovir for seven days (Figure 2). CBC showed: WBC: 6.45 with Neutrophils $\%=71$, Hemoglobin $=7.9$ and Platelets $=219$. Plain chest X-rays were normal. Abdominopelvic Computed Tomography scan (CT scan) revealed multiple hypodense masses in liver, spleen and pancreas, and one large mass with septations in left psoas muscle. Positive inguinal, aortocaval, retrocaval and para-aortic lymphadenopathy with central low attenuation (Figure 3). ATB was high on differential diagnosis for which Mantoux skin test was 
placed and found negative with $0 \mathrm{~mm}$ induration. Fine-needle aspiration (FNA) biopsy of Left psoas muscles resulted in a positive Acid-Fast Bacilli (AFB) smear and culture for Non-resistant Mycobacterium tuberculosis. Sputum and fecal AFB smear and cultures were negative. Anti-TB treatment was started, including Rifampin, Isoniazid, Ethambutol and Pyrazinamide (RIPE). HAART was changed to Emcitrabine-Dolutegravir and Tenofovir disoproxil. Patient remained in isolation for two weeks while on Direct Observed therapy. FNA was repeated after two weeks of prior mentioned treatment and found negative. Subsequently, isolation was discontinued and patient was discharged home. She was advised to complete six months of RIPE therapy plus Pyridoxine and follow up with Primary Care Physician (PCP) preventively to monitor drugs adverse effects.

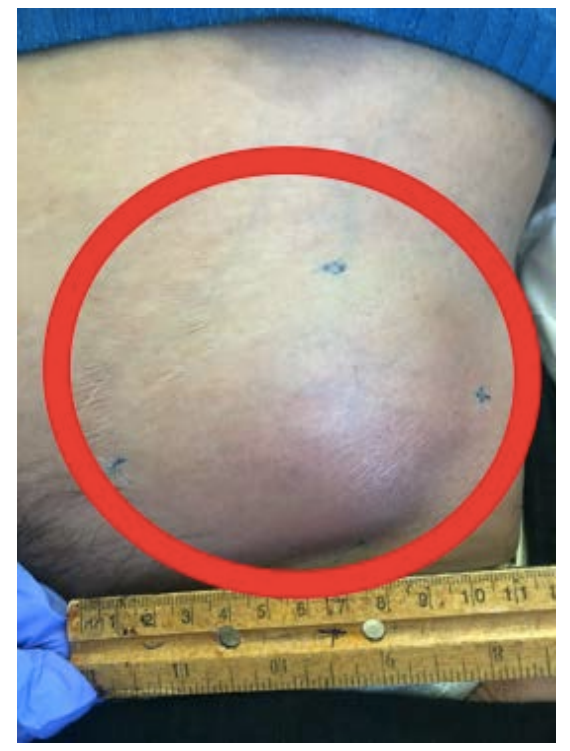

Figure $1.10 \mathrm{~cm} \times 6 \mathrm{~cm}$ non-mobile, violaceous mass.

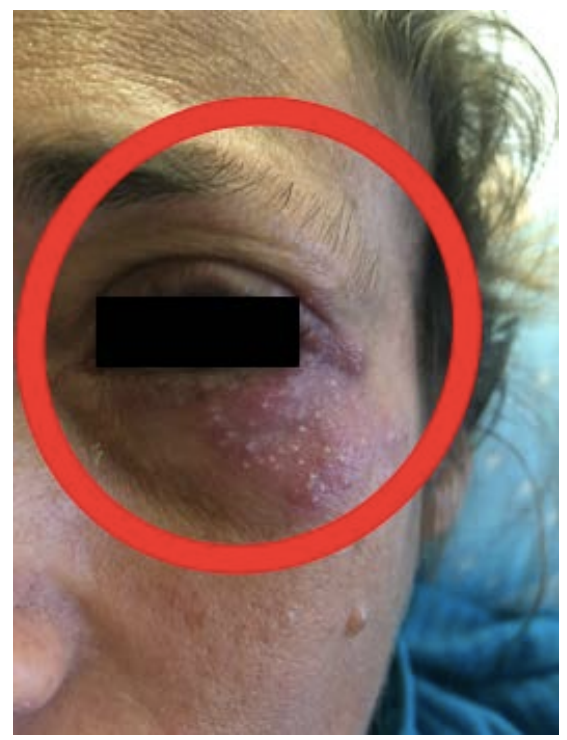

Figure 2. Left eye Herpetic zoster lesions. 


\section{Discussion}

The abdominal tuberculosis (ATB) occurs in four forms: Tuberculous lymphadenopathy, Peritoneal tuberculosis, Gastrointestinal tuberculosis (GITB) and Visceral tuberculosis involving solid organs [5]. A combination may occur as well. Presentation depends on the mechanism of bacterial entrance. The bacteria may enter through ingestion of infected sputum, manifesting as GITB. It may cause ulceration of mucosa that can spread through peritoneum, causing Peritoneal Tuberculosis. If active or latent pulmonary TB is present, it can spread through lymph nodes subsequently manifesting as Tuberculous lymphadenopathy. If bacilli enter through portal circulation, hematogenous spread to visceral organs may occur [5]. The most common site of GITB is the ileocecal region since it has minimal digestive activity, relative increased physiological stasis, higher rates of fluid and electrolyte absorption, as well as more lymphoid tissue [8]. While among the solid organs, liver and spleen presented the highest incidence of involvement [9]. Most common complications of ATB are intestinal obstruction secondary to strictures or adhesion, as well as perforation due to reactive fibrosis of peritoneum [10] [11].

Diagnosis is made through radiological studies including CT scan, US, Barium studies or MRI. CT scan is helpful to evaluate the extent and type of ATB. There are no pathognomonic radiological findings of ATB [6]. A normal liver on CT scan does not rules out hepatic TB because a miliary pattern may not be seen on CT scan. On CT scan, necrotic lymph nodes, which results from avascular caseating granulomatous lesion, in combination with hypoattenuating nodules on solid organs is highly sensitive for ATB [12]. If high bilirubin is seen in a highrisk/immunosuppressed patient living on endemic area of TB, hepatic biopsy should be performed to evaluate for small granulomas of miliary hepatic TB [13]. Stool cultures for tubercle bacilli are not recommended as a single test for diagnosis of GITB because positive results are more likely to occur in patients with active pulmonary disease who are swallowing the sputum, rather than in all patients with ATB [14]. Additionally, immunological test, such as Tuberculin skin test (TST) and IFN-Gamma Release Assay (IGRA) cannot distinguish between latent and active infection, and negative results cannot completely exclude the disease [14].

Psoas muscle abscess usually results from continuous Tuberculous spondylitis [12]. In our patient, the culprit of the left psoas muscle abscess was likely related to latent pulmonary TB spreading to lymph nodes with subsequent rupture of the left para-aortic lymph node within the muscle's fibers (Figure 4). The decision to perform the biopsy on the Left psoas muscle was based on easier accessibility when compared to inguinal or retroperitoneal lymphadenopathy. Accessibility to sampling area is an issue to be taken into consideration when diagnosing ЕРТВ. There are no preferred areas for sampling nor diagnostic algorithms, but the most accessible area to secure an adequate sample is the recommended area [15]. 

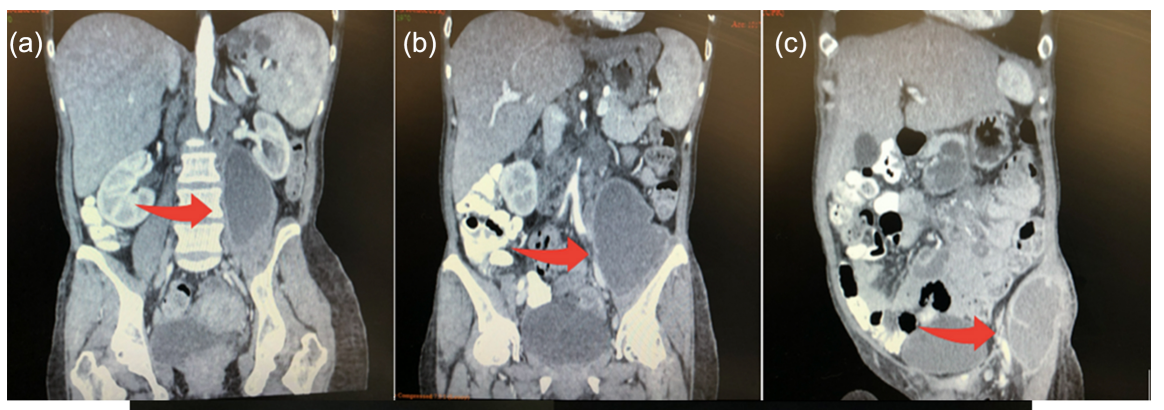

(d)

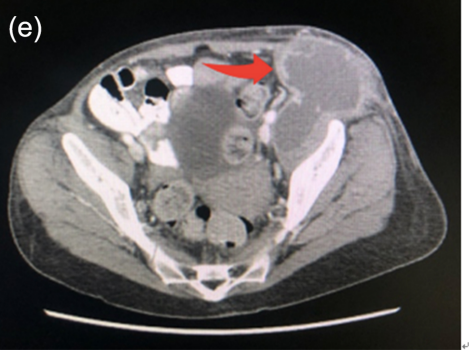

Figure 3. Abdominal CT scan coronal plane: (a)/(b): Cystic mass of the Left psoas muscle with extension to the iliacus muscle. (c) Extending below the levels of the inguinal ligament. Abdominal CT scan axial plane: (d) Psoas muscle mass (e) Extension to the iliacus muscle.

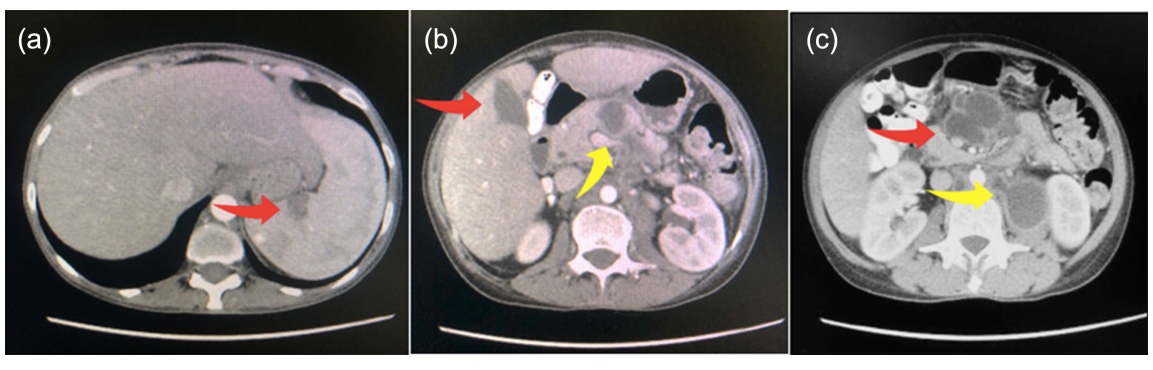

Figure 4. Abdominal CT scan Axial plane: (a) Spleen hypo-enhancing lesion in the posterior medial aspect of the pole (Red arrow). (b) Subdiaphragmatic hypodense lesion in the $\mathrm{R}$ hepatic lobe (Red arrow). Peripherally enhancing cystic/necrotic mass in the inferior portion of the pancreas (Yellow arrow). (c) Necrotic mesenteric lymphadenopathy (Red arrow). Left para-aortic lymphadenopathy (Yellow arrow).

New studies suggest that cases of ATB should meet the following criteria for diagnosis: Conventional positive AFB smear and culture for M. tuberculosis, Histopathological diagnosis with identification of caseating granulomas in biopsy and response to anti-tubercular treatment [7]. Six months of anti-tuberculous therapy for active ATB is generally considered adequate [16]. Young et al. (2008), reported considerable improvement in patients with intestinal TB at three months of therapy. Therefore, Korean guidelines recommend colonoscopy follow up to be performed every three months [17].

\section{Conclusion}

In the presented case, we emphasize that if a patient with HIV-AIDS complains of persistent abdominal pain or has FUO, ATB should be within the differential 
diagnosis. ATB has a varied clinical presentation and could be easily missed. Next steps in diagnosis should include abdominal CT scan looking for enlarged lymph nodes with center hypo-attenuation, parietal intestinal thickening, and miliary vs. macronodular hepatic pattern. Diagnosis should not be limited to radiological imaging but also include immunological test (TST and IGRA) of stool and sputum, AFB smear and culture, biopsy for histopathological diagnosis, body fluid analysis-Adenosine deaminase activity (ADA) - and/or Molecular tests-NAAT by PCR.

\section{Consent}

Verbal informed consent was obtained from the patient for this report, including pictures.

\section{Conflicts of Interest}

The authors declare no conflicts of interest regarding the publication of this paper.

\section{References}

[1] Chai, Q.Y., Zhan, Y. and Liu, C.H. (2018) Mycobacterium Tuberculosis: An Adaptable Pathogen Associated with Multiple Human Diseases. Frontiers in Cellular and Infection Microbiology, 8, 158. https://doi.org/10.3389/fcimb.2018.00158

[2] Singh, P., Kant, S., Gaur, P., Tripathi, A. and Pandey S. (2018) Extrapulmonary Tuberculosis: An Overview and Review of Literature. International Journal of LifeSciences Scientific Research, 4, 1539-1541.

[3] Khammissa, R.A.G., Wood, N.H., Meyerow, R., Lemmer, J., Raubenheimer, E.J. and Feller, L. (2011) Primary Oral Tuberculosis as an Indicator of HIV Infection. Pathology Research International, 2011, Article ID: 893295. https://doi.org/10.4061/2011/893295

[4] Kim, J.-H., Kim, E.S., Jun, K.-I., Jung, H.G., Bang, J.H., Choe, P.G., et al. (2018) Delayed Diagnosis of Extrapulmonary Tuberculosis Presenting as Fever of Unknown Origin in an Intermediate-Burden Country. BMC Infectious Disease, 18, Article No. 426. https://doi.org/10.1186/s12879-018-3349-5

[5] Debi, U., Ravisankar, V., Prasa, K.K., Sinha, S.K. and Sharma, A.K. (2014) Abdominal Tuberculosis of the Gastrointestinal Tract. World Journal of Gastroenterology, 20, 14831-14840. http://dx.doi.org/10.3748/wig.v20.i40.14831

[6] Sharma, M.P. and Bhatia, V. (2004) Abdominal Tuberculosis. Indian Journal of Medical Research, 120, 305-315.

[7] Awasthi, S., Saxena, M., Ahmad, F., Kumar, A. and Dutta, S. (2015) Abdominal TB: Diagnostic Dilemma. Journal of Clinical and Diagnostic Research, 9, EC1-EC3. https://doi.org/10.7860/JCDR/2015/13350.5887

[8] Paustian, F. and Marshall, J. (1985) Intestinal Tuberculosis. In: Edward Berk, J., Ed., Bockus Gastroenterology, WB Sunders, Philadelphia.

[9] Rocha, E.L., Pedrassa, B.C., Bormann, R.L., Kierszenbaum, M.L., Torres, R.L. and D'lppolito, G. (2015) Abdominal Tuberculosis: A Radiological View with Emphasis on Computed Tomography and Magnetic Resonance Imaging Findings. Radiologia Brasileira, 48, 181-191. https://doi.org/10.1590/0100-3984.2013.1801 
[10] Kapoor, V.K. (1998) Abdominal Tuberculosis. Postgraduate Medical Journal, 74, 459-467. https://doi.org/10.1136/pgmj.74.874.459

[11] Dasgupta, A., Singh, N. and Bhatia, A. (2009) Abdominal Tuberculosis: A Histopathological Study with Special Reference to Intestinal Perforation and Mesenteric Vasculopathy. Journal of Laboratory Physicians, 1, 56-61. https://doi.org/10.4103/0974-2727.59700

[12] Rodriguez, S.Y., Renjifo, M.E. and Medina, F.J. (2019) Extrapulmonary Tuberculosis: Pathophysiology and Imaging Findings. RadioGraphics, 39, 2023-2037. https://doi.org/10.1148/rg.2019190109

[13] Abu-Zidan, F. and Sheek-Hussein, M. (2019) Diagnosis of Abdominal Tuberculosis: Lessons Learned over 30 Years: Pectoral Assay. World Journal of Emergency Surgery, 14, Article No. 33. https://dx.doi.org/10.1186\%2Fs13017-019-0252-3

[14] Lee, J.Y. (2015) Diagnosis and Treatment of Extrapulmonary Tuberculosis. Tuberculosis and Respiratory Diseases, 78, 47-55. https://doi.org/10.4046/trd.2015.78.2.47

[15] Purohit, M. and Mustafa, T. (2015) Laboratory Diagnosis of Extra-Pulmonary Tuberculosis (EPTB) in Resource-Constrained Setting: State of the Art, Challenges and the Need. Journal of Clinical and Diagnostic Research, 9, EE1-EE6. https://doi.org/10.7860/JCDR/2015/12422.5792

[16] Ben-Ayed, H., Koubaa, M., Marrakchi, C., Rekik, K., Hammami, F., Smaoui, F., et al. (2018) Extrapulmonary Tuberculosis: Update on the Epidemiology, Risk Factors and Prevention Strategies. International Journal of Tropical Diseases, 1, 1-6. https://doi.org/10.23937/ijtd-2017/1710006

[17] Young, S.P., Dae, W.J., Seong, H.K., Han, H.L., Yun, J.J., Moon, H.S., et al. (2008) Colonoscopy Evaluation after Short Term Anti-Tuberculosis Treatment in NonSpecific Ulcers on the Ileocecal Area. World Journal of Gastroenterology, 14, 5051 5058. http://dx.doi.org/10.3748/wig.14.5051 